\title{
The Quality of Life of Women with Cervical Cancer in Indonesia: A Cross-Sectional Study
}

\author{
Yati Afiyanti ${ }^{1}$, Ice Yulia Wardani ${ }^{1}$, Evi Martha ${ }^{1}$ \\ ${ }^{I}$ Faculty of Nursing, University of Indonesia, Depok, Indonesia \\ Corresponding Author: Yati Afiyanti (yatikris@ui.ac.id)
}

\begin{abstract}
Background: Cervical cancer have significant impacts on the quality of life (QOL) of the women experiencing it. Limited studies are exploring QOL among Indonesian women diagnosed with cervical cancer.

Purpose: This study aimed to investigate the QOL of women with cervical cancer who received radiation therapy and chemotherapy.

Methods: A cross-sectional study was conducted to evaluate the QOL among 164 women with cervical cancer undergoing treatment. A consecutive sampling technique was used to recruit the samples. The EORTC QLQ-C 30 and QLQ-CX 24 were used to measure the QOL and sexual-related symptoms. The data collected were analyzed and presented as descriptive statistics in the form of means and standard deviations.

Results: The mean of the global health status of general well-being and QOL of the women was $54.56 \pm 25.47$. In the QOL subgroups of functional status, the physical function (55.94) and role functions (55.34) were most frequently reported to affect QOL. Regarding sexual-related symptoms of the women, sexual worry (52.72) and menopause symptoms (48.03) were the two most prevalent symptoms which impacted QOL.

Conclusions: The quality of life of women with cervical cancer in Indonesia was generally at the higher end of the scale, and yet the majority of the women reported high prevalent symptoms of cancer and adverse effects of treatments. Comprehensive, high quality and culturally sensitive care for women with cervical cancer needs to be implemented in Indonesia, which has unique social and cultural beliefs.
\end{abstract}

Keywords: Cervical cancer; quality of life; sexual and sexuality; women

How to Cite: Afiyanti, Y., Wardani, I. Y., Martha, E. (2019). The quality of life of women with cervical cancer in Indonesia: A cross-sectional study. Nurse Media Journal of Nursing, 9(2), 128-140. doi:10.14710/nmjn.v9i2.26014

Permalink/DOI: https://doi.org/10.14710/nmjn.v9i2.26014

\section{BACKGROUND}

Cervical cancer is one of the most prevalent cases among cancer cases in women. Cervical cancer significantly impacts the lives of women and their quality of life as its symptoms and treatment affect their physical, mental, and social well-being. The mainstream cancer treatment, including chemotherapy and radiotherapy, have a range of 
adverse effects which adds to the physical and psychological burden of the patients and families (Wenzel, Vergote, \& Cella, 2003). An assessment is, therefore, necessary to determine the level of impact on women's well-being. One of the most referred indicators is the quality of life of women with cervical cancer.

Quality of Life (QOL) has always been an important goal in the decades of developing the treatment of cancer. In women with cervical cancer, concerns such as the changes in body image and emerging physical problems because of the illness symptoms may lead to psychological problems. Over the years, many studies have been conducted to explore the quality of life of women with cervical cancer as well as its influencing factors (Garssen, Vos, de Jager Meezenbroek, de Klerk, \& Visser, 2011; Vistad, Fossa, $\&$ Dahl, 2006). It can be concluded that treatments of cancer may decrease the quality of life in patients despite the many options of treatments available in the present years. A study also proved that the quality of life of cancer patients was also influenced by other factors such as economic background, social status, and disease-related factors (Pradjatmo, Nisman, \& Fatmawati, 2017).

Another aspect of cervical cancer patient's well-being is the sexual-related symptom, i.e., sexuality and sexual activities. Cervical cancer profoundly affects the reproductive system of women, often resulting in sexual dysfunction. Vaginal dryness, stenosis, and dyspareunia have been reported among survivors who had undergone surgery, radiation, and chemotherapy (Huffman, Hartenbach, Carter, Rash, \& Kushner, 2016). It is an existing problem in women with cervical cancer, which can negatively affect the general quality of life.

Presently, studies of quality of life of women with cancer in Indonesia are still limited in number (Afiyanti, Martha, Wardani, \& Andrijono, 2018; Afiyanti, Milanti, \& Putri, 2018). On the other hand, according to the available information, Indonesia is one of the developing countries in Asia with a high incidence and mortality rate of cervical cancer cases (Ministry of Health Republic of Indonesia, 2015). Studies about quality of life are vital as evidenced where the improved quality of life of the cancer patients during the treatment process may increase patients' adherence to treatment and help them to remain strong in overcoming their symptoms (Perwitasari et al., 2011). Further research on the topic of quality of life of cervical cancer patients in Indonesia is urgently needed.

\section{PURPOSE}

This study aimed to investigate the quality of life among women with cervical cancer in Indonesian undergoing radiation therapy and chemotherapy.

\section{METHODS}

\section{Design and samples}

This study employed a descriptive cross-sectional research design. The sample size was calculated using the formula for descriptive studies with the aid of OpenEpi Version 3.0, an open-source calculator. The sample size generated was 164. Participants were selected through a consecutive sampling method. The study recruited 182 women with cancer undergoing treatment between April to August 2015. Of the 182 women 
recruited, 10 of the women were patients of other types of cancer. One hundred and seventy-two women were eligible for the study, of which, three women declined to participate, and 5 participants did not finish the questionnaire, resulting in 164 participants (95\% response rate).

The eligibility criteria were: (1) women diagnosed with cervical cancer; (2) under the age of 60 years, (4) able to read and write; (4) undergoing chemotherapy and radiation treatment in the gynecology oncology ward and clinic in the respective hospitals, and (5) expressed willingness to participate in the study. The participants were approached by the research team with permission from respective hospitals. Participants were provided relevant information about the study before signing the informed consent.

The study was conducted in three different hospitals with a gynecology oncology ward and outpatient clinic in different provinces in Indonesia, namely Bandung Hasan Sadikin Hospital in West Java, Margono Hospital in Central Java, and Wahidin Sudiro Husodo Hospital in Makassar, South Sulawesi.

\section{Ethical consideration}

The research was submitted to the Institutional Review Board, Research Ethics Committee of the Faculty of Medicine and the Faculty of Nursing, Universitas Indonesia, to obtain permission and had been approved (No.525/UN2.F1/ETIK/2015 and No. 0318/UN2.F12D/HKP.02.04/2015). All participants have given their informed consent prior to their participation in this study. This study adhered to the ethical principles of research, including the right to self-determination, patients' anonymity, and confidentiality. All participants' data were handled with the strictest confidence.

\section{Research instrument and data collection}

The participants were asked to complete two sets of questionnaires. The first questionnaire was social demographic information developed by the researchers. Sociodemographics data identified in the study were age, work status, educational level, marital status, and stage of disease based on the FIGO (Fédération Internationale de Gynécologie et d'Obstétrique) classification determined by the International Federation of Gynecology and Obstetrics. For cervical cancer, the staging started from stage IA to IVB with no stage 0 . The second questionnaire was the instrument to assess participants' quality of life. It consisted of 2 different parts: European Organization of Research and Treatment of Cancer Quality of Life Q-30 (EORTC QLQ-C30) and the Quality of Life Questionnaire Module Cervical Cancer (EORTC QLQ-CX24).

The EORTC QLQ-C30 consists of 30 questions with 28 questions in a four-point Likert-style evaluation and two questions (q29 and q30) evaluated through a scale of 1 to 7 ( $1=$ very bad, $7=$ xcellent. The scale assesses five functionality dimensions, i.e., physical, role, emotional, cognitive, and social functions, as well as several prevalent symptoms. A high score for the global health status/QOL represents a high QOL. Similarly, a high score for functional status in the scale represents a high/healthy level of functioning. On the contrary, a high score for a symptom item indicates a high level of symptoms/problems. 
The EORTC QLQ-CX24 has 24 questions, which are divided into eight sectors. The questionnaire was specifically used to identify the symptoms experienced by women with cervical cancer in relation to their sexuality and sexual activities. Similarly, each section of the EORTC QLQ-CX24 provides a summary score indicating the degree of the problems in the particular section.

Both questionnaires' validity and reliability properties have been evaluated in prior studies (Perwitasari et al., 2011; Hua et al, 2013). Cronbach's $\alpha$ coefficient of the domains was at 0.7-0.9, with no domain was below 0.7. After both questionnaires were translated into the Indonesian language, it was tested for readability. Also, the instrument was subjected to a pilot test followed by a review from the fellow lecturers to finalize the questionnaire. The questionnaires were given to the participants on papers and were completed by the participants independently. In average, participants took 15 to 30 minutes to complete all questionnaires

\section{Data analysis}

After data collection was completed, all data were analyzed using the Statistical Package for Social Sciences (SPSS) software version 17 (SPSS Inc., USA). Data were then analyzed and presented as descriptive statistics in the form of means and standard deviations.

\section{RESULTS}

The demographic characteristics of the respondents are presented in Table 1 . The mean age of women in this study was 48.83 years. Almost half of the respondents $(43.9 \%)$ completed elementary school as their highest education level, and $80.49 \%$ of the respondents were housewives and did not work. Most of the women were married $(81.10 \%)$, and more than half were diagnosed with stage II or stage III using the FIGO staging classification (Table 1).

Table 1. Demographic characteristics of the respondents

\begin{tabular}{|c|c|c|}
\hline Demographic characteristics: & Mean \pm SD & $\%$ \\
\hline Age & $48.83 \pm 10.01$ & \\
\hline \multicolumn{3}{|l|}{ Education Level } \\
\hline Elementary school & & 43.90 \\
\hline Junior high school & & 20.73 \\
\hline Senior high school & & 27.44 \\
\hline Bachelor & & 7.93 \\
\hline \multicolumn{3}{|l|}{ Work status: } \\
\hline Working & & 19.51 \\
\hline Housewife & & 80.49 \\
\hline \multicolumn{3}{|l|}{ Marital status: } \\
\hline Married & & 81.10 \\
\hline Widowed & & 18.90 \\
\hline
\end{tabular}




\begin{tabular}{|c|c|c|}
\hline Demographic characteristics: & Mean \pm SD & $\%$ \\
\hline \multicolumn{3}{|l|}{ Stage of Disease (FIGO) } \\
\hline Stage I & & 13.41 \\
\hline Stage II & & 46.95 \\
\hline Stage III & & 34.15 \\
\hline Stage IV & & 5.49 \\
\hline
\end{tabular}

Table 2 presents the mean score of general well-being and QOL (54.56). This study also showed that the women found their physical and role functions (55.94 and 55.34) were mostly affected by their cancer, followed by emotional functions (54.71), cognitive functions (48.79), and social functions (43.29) interference. The most frequently reported symptoms of sub-group were fatigue (69.93), anorexia (66.72), pain (66.11), financial difficulties (62.04), and sleeplessness (61.59).

Table 2. Quality of life index and subscale points $(n=164)$

\begin{tabular}{|c|c|c|c|}
\hline & Items & $\begin{array}{l}\text { Reference } \\
\text { Value* }\end{array}$ & Mean \pm SD \\
\hline \multicolumn{4}{|l|}{ Quality of life Subscales Points: } \\
\hline General well-being \& quality of health & 29,30 & $\mathrm{~N} / \mathrm{A} * *$ & $54.56 \pm 5.47$ \\
\hline \multicolumn{4}{|l|}{ Functional Status: } \\
\hline Physical functions & $1,2,3,4,5$ & N/A & $55.94 \pm 19.58$ \\
\hline Role functions & 6,7 & N/A & $55.34 \pm 25.97$ \\
\hline Emotional functions & $21,22,23$ & 64.2 & $54.71 \pm 22.27$ \\
\hline Cognitive functions & 20,25 & 86.0 & $48.79 \pm 20.18$ \\
\hline Social functions & 26,27 & 77.1 & $43.29 \pm 22.38$ \\
\hline \multicolumn{4}{|l|}{ Symptoms: } \\
\hline Fatigue & $10,12,18$ & 33.8 & $69.93 \pm 21.37$ \\
\hline Nausea and vomiting & 4,15 & 8.7 & $58.81 \pm 21.33$ \\
\hline Pain & 9,19 & 29.4 & $66.11 \pm 23.91$ \\
\hline Difficulty in breathing & 8 & 16.2 & $40.06 \pm 23.33$ \\
\hline Sleeplessness & 11 & 36.6 & $61.59 \pm 28.28$ \\
\hline Anorexia & 13 & 15.9 & $66.72 \pm 26.59$ \\
\hline Constipation & 16 & 20.2 & $51.51 \pm 26.50$ \\
\hline Diarrhea & 17 & 7.1 & $40.21 \pm 21.63$ \\
\hline Symptoms Economic difficulties & 28 & 9.9 & $62.04 \pm 27.21$ \\
\hline
\end{tabular}

*Reference value was based on EORTC QLQ-C30 Reference Values by the EORTC Groups (2008)

**Not available, reference number was based on the updated questionnaire

The data collected with the QLQ-CX24 showed that sexual worry (52.72) was the most reported problem, followed by menopause symptoms (48.03) as the second and lymphedema (46.89) as the third commonest symptom (See Table 3). 
Table 3. EORTC QLQ-CX24 mean points $(n=164)$

\begin{tabular}{lll}
\hline & Items & Mean \pm SD \\
\hline Symptoms experience & $1,2,3,4,5,6,7,9,11,12,13$ & $43.19 \pm 14.43$ \\
Body Image & $15,16,17$ & $46.41 \pm 21.09$ \\
Sexual functions & $20,21,22,23$ & $35.31 \pm 20.97$ \\
Lymphoedema & 8,10 & $46.89 \pm 19.04$ \\
Menopause symptoms & 14 & $48.03 \pm 25.30$ \\
Sexual worry & 18 & $52.72 \pm 30.99$ \\
Sexual activity & 19 & $33.78 \pm 18.88$ \\
Sexual enjoyment & 24 & $31.81 \pm 16.65$ \\
\hline
\end{tabular}

\section{DISCUSSION}

Gynecological cancer, namely ovarian, breast, and cervical cancer, dominate cancer incidence in Indonesian women. For cervical cancer, Indonesia ranks second as the country with the highest number of new cervical cancer cases and ranks third for the most death by cervical cancer globally (The Global Cancer Observatory, 2019). The high incidence and mortality rate of cervical cancer in Indonesia ushered increased attention of studies related to the topic; however, many aspects of cervical cancer in Indonesia still need further research. The quality of life of cervical cancer patients is a critical aspect of the illness to study. The purpose of this study was to identify the quality of life of women diagnosed with cervical cancer who have undergone radiotherapy and chemotherapy.

The results of the EORTC QLQ-C30 showed the mean of general well-being and quality of life in this study was on the upper half of the scale (54.56 \pm 25.47$)$. Similarly, a higher value of global health status and quality of life were reflected in some studies in Asia (Akkuzu, 2012; Kumar et al., 2014; Lee et al., 2016). On the other hand, a large percentage of the participants (see Table 2) also reported symptoms, indicating a gap between the perceived quality of life and the reality of symptoms. This gap between the perceived quality of life and the reality of the symptoms experience is likely to be the result of several influences. Firstly, the lack of knowledge and understanding about cancer and its treatment may contribute to the higher value of general well-being and quality of life. Knowledge and understanding directly impact the level of awareness. Other studies reported varying results of the association of the level of awareness with the value of quality of life of cancer patients. Some cancer patients with a strong awareness of disease status and progression showed a positively higher perceived quality of life compared to the patients with low awareness (Lee et al., 2013; Talepasand, Pooragha, \& Kazemi, 2013). While another study found higher awareness of disease prognosis in terminally ill cancer patients was associated with a lower value of quality of life (El-Jawahri et al., 2014).

Compared to European countries where cancer studies were extensive, Asian countries have relatively less exposure to the information and understanding of cancer and its treatment. In particular, knowledge and understanding of cancer were not well 
established within the general society in Indonesia. Many cancer patients believed completing treatment or finishing their primary treatment regimen meant they were healed, so patients stopped attending health care services. This behavior was also observed in a study to explore the reason for delaying seeking help and non-adherence to treatment in Indonesia. One of the reasons reported was the lack of awareness and knowledge (Iskandarsyah et al., 2014).

A large percentage of the women in this study were at stage II (46.95\%), and stage III $(34.15 \%)$ of cervical cancer and the women perceived a relatively high score of general well-being and quality of health. The study was conducted towards women with cervical cancer at the end of their treatment. The researcher determined the timing of participation of the respondent factored in the higher level of general well-being and quality of health depicted. Previous quality of life studies in cervical cancer patients reported similar findings. Patients with an advanced case of cervical cancer had higher QOL scores after the treatment was undertaken (Dehkordi, Heydarnejad, \& Fatehi, 2009; Dahiya et al., 2016). Patients nearing the end of their treatment regimen would have undergone a considerable amount of treatment and perceived better health and a positive experience compared to before they received treatment.

Another factor that is likely to influence the higher score of QOL was a shift in values or beliefs. Sprangers and Schwartz (1999) presented the idea of response shift integration into health-related quality of life, and the concept has been a subject of research in the following years. Serious illnesses such as cervical cancer would likely affect the patient's internal standards, values, and perception and created a change to what is regarded as important in their lives. The change shifted their priorities, values, and beliefs to better their ability to cope with the consequences of the illness and its treatment. The patients would present a more positive attitude towards the illness and perception of their health status.

This study found a gap between the perceived quality of life and the reality of the symptoms experienced, meaning that the QOL mean value might not necessarily reflect the symptoms experienced by the women. The participants of this study reported varying physical problems during the continuum of cancer and care with fatigue, anorexia, sleeplessness, pain, nausea and vomiting, and constipation as the most reported symptoms. The symptoms reported in this study coincides with the findings in several similar studies of quality of life in cervical cancer patients (Clevenger et al., 2013; Endarti et al., 2015). It demonstrated that treatment did not eliminate the physical discomfort of patients and may also exacerbate particular symptoms despite a higher score of the general well-being and quality of health of patients.

Furthermore, compared to the earlier studies, the researcher noted that the mean of the symptoms score reported by participants was significantly higher than the studies with a similarly higher score of general well-being and functional status. A study on the quality of life of women with cervical cancer in Taiwan presented a mean value of 62.69 for Global health status/QOL score and 95.39 for physical functioning, while fatigue and pain symptoms score were 5.95 and 3.99, respectively (Li, Chang, Tsai, \& Chen, 2017). 
Another study in Indonesia similarly showed a higher score of general well-being/QOL and symptoms subscale scores on the lower end of the range (Dahiya et al., 2016).

In addition to the score of general well-being and quality of health which was in the higher end of score range, several functional statuses were also on the higher end of the score range. Physical and role functions were on the higher end of the score, while cognitive and social functions were on the lower end of the score range. Such distribution of mean was expected to happen as the majority of the quality of life studies with the EORTC QLQ CX-30 instrument had shown a similar trend of distribution. The emotional, cognitive, and social functions score in this study was, however, much lower compared to other studies with social functions as the lowest score (Akkuzu, 2012; Torkzahrani, Rastegari, Khodakarami, Akbarzadeh-baghi-, \& Alizadeh, 2013; Dahiya et al., 2016; Lee et al., 2016; Li et al., 2017). Cervical cancer and treatment are a considerable burden on the patients' body. This presented limitations to patients' ability to complete activities they were used before the experience. Such limitation is likely to cause distress in patients and interfere with the way they interact with their surroundings. In Indonesia, where the general society is comparatively more conservative, illness such as cervical cancer has a debilitating impact on the social life of a patient. The stigma surrounding cancer and its patient, as well as the negative cultural beliefs of cancer, prevented patients from resuming their normal social functions (Iskandarsyah et al., 2014).

In the current study, a significant presence of economic difficulties was also observed. Most participants in this study were housewives with no source of income and were entirely dependent on their partners. Presently in Indonesia, the Universal Health Coverage program from the Indonesian government covered the cost of treatment for cancer, thus eliminating a vital part of the economic burden. However, expenses such as transportation, family expenses during the patient's care period, and other miscellaneous expenses must be covered by the patients and their families themselves, amounting to a considerable number. As a reflection, a study in Europe reported of the total cost of cancer in 2009, $40 \%$ of the cost was for health care while the remaining was accounted for informal care cost and other costs (Luengo-Fernandez, Leal, Gray, \& Sullivan, 2013). Similarly, economic difficulties were reported in previous studies exploring the quality of life of patients with cervical cancer and gynecological cancer in general (Fathollahzade et al., 2015; Ogoncho, Omuga, Wakasiaka, \& Muiva, 2015; Pradjatmo et al., 2017; Sharp \& Timmons, 2010). The presence of health insurance covering the health care cost of cancer treatment, thus assisted the patient and family to cope with the economic cost of cancer. However, other costs, such as informal care cost and other non-health related cost, was still a considerable financial burden.

This study employed the EORTC QLQ-CX24 to identify the symptoms experienced by women with cervical cancer in relation to their sexuality and sexual activities. The result showed that sexual worry was most prevalent reported by the women, followed by menopause symptoms, lymphedema, and body image.

In patients with gynecological cancer, including cervical cancer, sexuality concerns are a vital aspect of their quality of life. Cervical cancer itself causes sexual-related 
concerns in patients, such as concern about their sexual performance, functionality, and activity. The radiotherapy and chemotherapy have been known to cause dyspareunia, decreased lubrication, and dryness, which added to the patient's anxiety about their sexual performance. These accumulated to sexual worry in patients diminishing their confidence to engage in sexual activity. Similar results were observed in other studies (Barnas, Skret-Magierio, Skret, \& Bidzinski, 2012; Kumar et al., 2014).

The mean value of lymphedema in this study was among the highest five values aside from sexual worry, menopause symptoms, body image, and symptoms experienced. Other studies noted similar findings where a significant number of the participants reported lymphedema related symptoms (Frøding, Ottosen, Mosgaard, \& Jensen, 2015; Lee et al., 2016). In patients with cervical cancer, treatment modalities, including chemotherapy and radiotherapy in the pelvic area, exacerbates the symptoms of lymphedema. Prolonged or recurring lymphedema related symptoms negatively affect the quality of life of women with cervical cancer.

Furthermore, this study found that concerns about body image were among the most prevalent. Body image is a part of the quality of life. A disturbed body image could cause emotional distress in women. The women participating in this study have undergone radiotherapy and chemotherapy. Cervical cancer patients undergoing radiotherapy and chemotherapy commonly experience vaginal changes such as dryness, lack of lubrication, dyspareunia, and sexual dysfunction. These changes could lower women's self-esteem and motivation to engage in sexual activities. In Indonesia, the cultural and social environment of women dictated sexual activities as a duty to their partners, and failure in fulfilling this duty equates a failure as a woman (Afiyanti \& Milanti, 2013). It brought looped feedback to the women's sense of her body and further lowered their sexual feelings and sense as a woman (feminine identity). Failure to identify the problem could interfere with the women's role in her family and the social role in the perception shared by the majority of Indonesian.

In the cultural belief of many Indonesian women, women are sexually functional and have fulfilled their duty when they can satisfy their partners, unrelated to any problem they faced to perform this duty (Afiyanti \& Milanti, 2013). Sexual problems often remain undetected due to the restriction of such cultural beliefs. Menopausal symptoms were presented to be on the higher end of the score range in this study; however, few women had reported them as indicated in the low value of the sexual function in the EORTC QLQ-CX24 section. Other studies in different countries have compared the sexual function in women with a cancer history and women with no cancer history, and similarly found menopausal symptoms did not reflect in sexual functioning. There was no observed difference between the interaction of menopause problems with sexual functioning in cancer patients and women with no cancer history (Marino et al., 2014; Lee et al., 2016).

The present study findings have some limitations. Despite being a multicenter study, the convenient sampling and relatively small sample size limit the generalizability of the study. Furthermore, on the characteristic respondents, this study did not assess the duration of illness of respondents, which also contributes to respondents' quality of life. 
As a result, the researchers were not able to determine a change in QOL of the women through the duration of illness. This study also presented limited evidence about the sexuality of women with cervical cancer as the questionnaire employed in this study mainly focused on the activity and functional aspect of sexuality. Sexuality is a complex concept, and a broader approach in research is needed to explore the concept of cervical cancer patients further. Regardless, this study presented evidence of the impact of cervical cancer, its treatment, and the adverse effects of the treatment on the quality of life of women with cervical cancer. Perceived general well-being and quality of life were not a reflection of the actual symptoms reported. This study recommends providing a more comprehensive assessment to offer a factual description of the women's quality of life and their actual symptoms experience.

\section{CONCLUSION}

In conclusion, the quality of life of women with cervical cancer in Indonesia was generally at the higher end of the scale. However, most participants also reported experiencing many symptoms as expressed in the high score on the symptoms scale. Assessing the quality of life of women with cervical cancer is needed by nurses and other health care professionals to develop patient care plans. Currently, cervical cancer treatment still causes considerable discomfort for patients, as reflected in the study results. Thus, formulating a more comprehensive care plan and providing more options for supportive care is necessary to improve the treatment experience of patients undergoing therapy as well as meeting their needs. A culturally sensitive design might benefit more to be implemented in Indonesia with its unique social and cultural beliefs.

\section{ACKNOWLEDGMENT}

This study was conducted with the financial support of the Directorate of Research and Community Engagement of the University of Indonesia through 2015 National Collaboration Grants with reference number: No: 1799/UN2.R12/HKP.05.00/2015. The funding source did not influence the overall research process, results from interpretation, and all the following subsequence process. The authors sincerely express gratitude to all participants of this research.

\section{CONFLICT OF INTEREST}

The authors declare no conflict of interest in this study.

\section{REFERENCES}

Afiyanti, Y., Martha, E., Wardani, I. Y., \& Andrijono, A. (2018). Development of supportive care to increase the quality of life of the gynecological cancer survivors in Indonesia. (Unpublished manuscript). Universitas Indonesia, Depok, Indonesia.

Afiyanti, Y., \& Milanti, A. (2013). Physical sexual and intimate relationship concerns among Indonesian cervical cancer survivors: A phenomenological study. Nursing \& Health Sciences, 15(2), 151-156. doi:10.1111/nhs.12006

Afiyanti, Y., Milanti, A., \& Putri, R. H. (2018). Supportive care needs in predicting the quality of life among gynecological cancer patients. Canadian Oncology Nursing Journal, 28(1), 1-26. 
Akkuzu, G. (2012). Quality of life of women undergoing chemotherapy for a gynaecological oncological disease in Turkey. Asian Pacific Journal of Cancer Prevention, 13(4), 1277-1280. doi:10.7314/APJCP.2012.13.4.1277

Barnas, E. Skret-Magierio, J., Skret, A., \& Bidzinski, M. (2012). The quality of life of women treated for cervical cancer. European Journal of Oncology Nursing, 16, 59-63.

Clevenger, L., Schrepf, A., DeGeest, K., Bender, D., Goodheart, M., Ahmed, A., ... \& Mendez, L. (2013). Sleep disturbance, distress, and quality of life in ovarian cancer patients during the first year after diagnosis. Cancer, 119(17), 3234-3241. doi:10.1002/cncr.28188

Dahiya, N., Acharya, A. S., Bachani, D., Sharma, D. N., Gupta, S., Haresh, K. P., \& Rath, G. K. (2016). Quality of life of patients with advanced cervical cancer before and after chemoradiotherapy. Asian Pacific Journal of Cancer Prevention, 17(7), 3095-3099. doi:10.14456/apjcp.2016.59/APJCP.2016.17.7. 3095

Dehkordi, A., Heydarnejad, M. S., \& Fatehi, D. (2009). Quality of life in cancer patients undergoing chemotherapy. Oman Medical Journal, 24(3), 204-208. doi:10.5001/omj.2009.40

El-Jawahri, A., Traeger, L., Park, E. R., Greer, J. A., Pirl, W. F., Lennes, I. T., ... \& Temel, J. S. (2014). Associations among prognostic understanding, quality of life, and mood in patients with advanced cancer. Cancer, 120(2), 278-285. doi: $10.1002 /$ cncr. 28369

Endarti, D., Riewpaiboon, A., Thavorncharoensap, M., Praditsitthikorn, N., Hutubessy, R., \& Kristina, S. A. (2015). Evaluation of health-related quality of life among patients with cervical cancer in Indonesia. Asian Pacific Journal of Cancer Prevention, 16(8), 3345-3350. doi:10.7314/APJCP.2015.16.8.3345

Fathollahzade, A., Rahmani, A., Dadashzadeh, A., Gahramanian, A., Esfahani, A., Javanganji, L., \& Nabiolahi, L. (2015). Financial distress and its predicting factors among iranian cancer patients. Asian Pacific Journal of Cancer Prevention, 16(4), 1621-1625. doi:10.7314/APJCP.2015.16.4.1621

Frøding, L. P., Ottosen, C., Mosgaard, B. J., \& Jensen, P. T. (2015). Quality of life, urogynecological morbidity, and lymphedema after radical vaginal trachelectomy for early-stage cervical cancer. International Journal of Gynecologic Cancer, 25(4), 699-706.

Garssen, B., Vos, P., de Jager Meezenbroek, E., de Klerk, C., \& Visser, A. (2011). Analyzing differences between psychotherapy groups and social support groups for breast cancer patients: development of an assessment method using video recordings. Patient Education and Counseling, 82(3), 377-383. doi:10.1016/j.pec.2010.11.018

Hua, C. H., Guo, H. M., Guan, X. L., Kong, F. J., Hou, R. J., Zhang, X. Y., \& Li, S. R. (2013). Validation of the European Organization for Research and Treatment of Cancer cervical cancer module for Chinese patients with cervical cancer. Patient Preference and Adherence, 7, 1061-1066. doi:10.2147/PPA.S52498

Huffman, L. B., Hartenbach, E. M., Carter, J., Rash, J. K., \& Kushner, D. M. (2016). Maintaining sexual health throughout gynecologic cancer survivorship: A comprehensive review and clinical guide. Gynecologic oncology, 140(2), 359368. doi:10.1016/j.ygyno.2015.11.010 
Iskandarsyah, A, de Klerk, C, Suardi, D.R, Soemitro, M.P, Sadarjoen, S.S, \& Passchier, J. (2014). Psychosocial and cultural reasons for delay in seeking help and nonadherence to treatment in Indonesian women with breast cancer: A qualitative study. Health Psychology, 33(3), 214-221. doi:10.1037/a0031060

Kumar, S., Rana, M. L., Verma, K., Singh, N., Sharma, A. K., Maria, A. K., ... \& Saini, S. (2014). PrediQt-Cx: post treatment health related quality of life prediction model for cervical cancer patients. PloS One,9(2), e89851. doi:10.1371/journal.pone.0089851

Lee, M. K., Baek, S. K., Kim, S. Y., Heo, D. S., Yun, Y. H., Park, S. R., \& Kim, J. S. (2013). Awareness of incurable cancer status and health-related quality of life among advanced cancer patients: a prospective cohort study. Palliative Medicine, 27(2), 144-154. doi:10.1177/0269216311429042

Lee, Y., Lim, M. C., Kim, S. I., Joo, J., Lee, D. O., \& Park, S. Y. (2016). Comparison of quality of life and sexuality between cervical cancer survivors and healthy women. Cancer Cesearch and Treatment: Official Journal of Korean Cancer Association, 48(4), 1321-1329.

Li, C. C., Chang, T. C., Tsai, Y. F., \& Chen, L. (2017). Quality of life among survivors of early-stage cervical cancer in Taiwan: an exploration of treatment modality differences. Quality of Life Research, 26(10), 2773-2782. doi:10.1007/s11136017-1619-0

Luengo-Fernandez, R., Leal, J., Gray, A., \& Sullivan, R. (2013). Economic burden of cancer across the European Union: a population-based cost analysis. The Lancet Oncology, 14(12), 1165-1174. doi:10.1016/s1470-2045(13)70442-x

Marino, J. L., Saunders, C. M., Emery, L. I., Green, H., Doherty, D. A., \& Hickey, M. (2014). Nature and severity of menopausal symptoms and their impact on quality of life and sexual function in cancer survivors compared with women without a cancer history. Menopause, 21(3), 267-274. doi:10.1097/gme.0b013e3182976f46

Ministry of Health Republic of Indonesia. (2015). Data dan informasi kesehatan situasi penyakit kanker [Data and information on cancer situation]. Buletin Kanker, 1(1), 1-5. doi:10.1007/s13398-014-0173-7.2

Ogoncho, I. M., Omuga, B. O., Wakasiaka, S., \& Muiva, M. (2015). Determinants of quality of life among gynaecological cancer patients on follow up at a referral hospital in Kenya. American Journal of Nursing Science, 4, 127-130. doi:10.11648/j.ajns.20150403.22

Perwitasari, D. A., Atthobari, J., Dwiprahasto, I., Hakimi, M., Gelderblom, H., Putter, H., ... \& Kaptein, A. A. (2011). Translation and validation of EORTC QLQ-C30 into Indonesian version for cancer patients in Indonesia. Japanese Journal of Clinical Oncology, 41(4), 519-529. doi:10.1093/jjco/hyq243

Pradjatmo, H., Nisman, W. A., \& Fatmawati, Y. (2017). Quality of life of cervical cancer patient with support from nuclear family and extended family in Dr. Sardjito general hospital, Yogyakarta Indonesia: A comparative study. International Journal of Research in Medical Sciences, 5(8), 3554-3559.

Sharp, L., \& Timmons, A. (2010). The financial impact of a cancer diagnosis. Ireland: National Cancer Registry Ireland/Irish Cancer Society. 
Sprangers, M. A., \& Schwartz, C. E. (1999). Integrating response shift into healthrelated quality of life research: A theoretical model. Social Science \& Medicine, 48(11), 1507-1515. doi:10.1016/s0277-9536(99)00045-3

Talepasand, S., Pooragha, F., \& Kazemi, M. (2013). Resiliency and quality of life in patients with cancer: moderating role of duration of awareness of cancer. Iranian journal of cancer prevention, 6(4), 222-226.

The Global Cancer Observatory. (2019). Indonesia. Retrieved from https://gco.iarc.fr/today/data/factsheets/populations/360-indonesia-fact-heets.pdf

Torkzahrani, S., Rastegari, L., Khodakarami, N., Akbarzadeh-Baghian, A., \& Alizadeh, K. (2013). Quality of life and its related factors among Iranian cervical cancer survivors. Iranian Red Crescent Medical Journal, 15(4), 320-323. DOI: 10.5812.ircmj.4410

Vistad, I., Fossa, S.D., \& Dahl. A.A., G. O. (2006). A critical review of patient-rated quality of life studies of long-term survivors of cervical cancer. Gynecologic Oncology, 102, 563-572.

Wenzel, L., Vergote, I., \& Cella, D. (2003). Quality of life in patients receiving treatment for gynecologic malignancies: Special considerations for patient care. International Journal of Gynecology \& Obstetrics, 83, 211-229. doi:10.1016/S0020-7292(03)90123-8 